\title{
Design and quantitative analysis of parametrisable eFPGA-architectures for arithmetic
}

\author{
B. Neumann, T. von Sydow, H. Blume, and T. G. Noll \\ Chair of Electrical Engineering and Computer Systems, RWTH Aachen University, 52062 Aachen, Germany
}

\begin{abstract}
Future SoCs will feature embedded FPGAs (eFPGAs) to enable flexible and efficient implementations of high-throughput digital signal processing applications. Current research projects on and emerging products containing FPGAs are mainly based on "standard FPGA"-architectures that are optimised for a very wide range of applications. The implementation costs of these FPGAs are dominated by a very complex interconnect network. This paper presents a method to improve the efficiency of eFPGAs by tailoring them for a certain application domain using a parametrisable architecture template derived from the results of a systematic evaluation of the requirements of the application domain.

Two different architectures are discussed, a reference architecture to illustrate the methodology and possible optimisation measures as well as a specialised arithmetic-oriented eFPGA for applications like correlators, decoders, and filters. For the arithmetic-oriented architecture, a novel logic element (LE) and a special interconnect architecture that was designed with respect to the connectivity characteristics of regular datapaths, are presented. For both architecture templates, physically optimised implementations based on an automatic design approach have been created.

As a first cost comparison of these implementations with standard FPGAs, the LE-density (number of logic elements per $\mathrm{mm}^{2}$ ) is evaluated. For the arithmetic-oriented architecture, the LE-density could be increased by an order of magnitude compared to standard architectures.
\end{abstract}

\section{Introduction}

The algorithmic complexity of current and future digital signal processing systems leads to an ever growing demand for performance, while short product cycles, mobility and

Correspondence to: B. Neumann

(neumann@eecs.rwth-aachen.de)

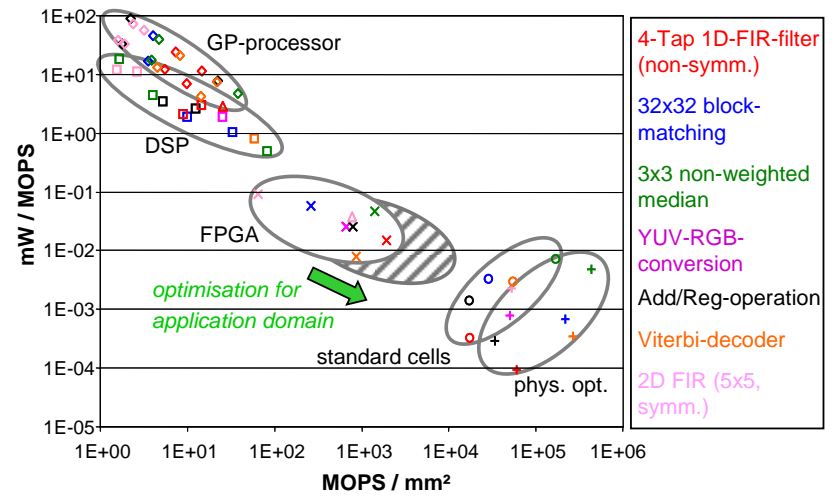

Fig. 1. Design space for different architectures.

cost-restrictions make the use of dedicated integrated circuits unfeasible. Fig. 1 illustrates the design space in terms of power efficiency (in mW/MOPS) and area efficiency (in MOPS $/ \mathrm{mm}^{2}$ ) for frequently used digital signal processing tasks implemented on different architectures like general purpose processors, Field Programmable Gate Arrays (FPGAs), physically optimised macros etc. As is well known from Fig. 1, programmable processor-architectures provide the lowest power- and area efficiency but feature highest flexibility. In contrast, physically optimised macros lead to the highest efficiency, but are inflexible. FPGAs offer an attractive compromise between these two extremes, as they allow for highly parallelised implementations while preserving insystem reconfigurability.

To meet the contrary demands of today's and future systems, different architecture blocks are integrated on a single chip building a heterogeneous System-on-a-Chip (SoC). A so-called SoC platform addresses a set of applications with similar requirements and characteristics (application domain). This offers the possibility to optimise the included components for this application domain. In the same way

Published by Copernicus GmbH on behalf of the URSI Landesausschuss in der Bundesrepublik Deutschland e.V. 


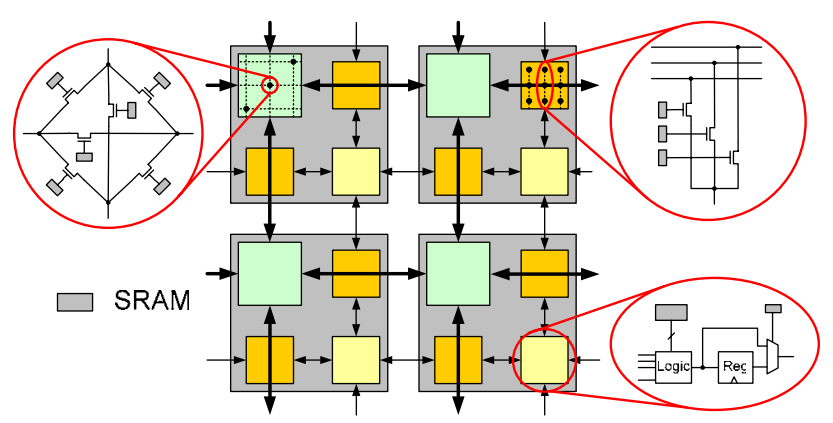

Fig. 2. General island-style FPGA-architecture.

the optimisation for an application domain improves the efficiency of a Digital Signal Processor (DSP) over a general purpose CPU, the architecture of an embedded FPGA (eFPGA) can be optimised by designing it for a dedicated application domain. The potential of improvement in efficiency for application domain specific eFPGAs was demonstrated for example in Neumann et al. (2003) and Leijten-Nowak et al. (2003). This paper focuses on arithmetic-oriented applications like correlators, decoders and filters, as they promise a high optimisation potential concerning all structural elements of an eFPGA-architecture.

The paper is structured as follows: In Sect. 2, a commonly applied architecture for FPGAs and its characteristics concerning area and power dissipation costs is sketched. The properties of eFPGAs specified for an arithmetic-oriented application domain are described in Sect. 3. In Sect. 4, the concept of parametrisable eFPGA-architectures is explained and two architecture templates with corresponding implementations are demonstrated. Conclusions are given in Sect. 5.

\section{FPGA-architectures}

Most common FPGA-architectures, as described e.g. in Brown et al. (1996), consist of three basic building blocks: Logic Elements (LE), Routing Switches (RS) and Connection Boxes (CB). Figure 2 shows the architecture of a socalled island-style FPGA-architecture as it was used in the first commercial devices. Most state-of-the-art FPGAs are still based on a modified island-style architectural concept.

An LE typically implements basic boolean functionality on bit-level. In most commercial FPGAs, the architecture of an LE is based on one or more lookup-tables (LUTs). A LUT with $N$ inputs shall be called LUT- $N$ in the following. Connection Boxes allow to connect interconnect lines either as in- or outputs of the corresponding LEs. The routing switch provides configurable connections between all its terminals to route signals through the FPGA. Most modern FPGAs use SRAMs to store the configuration information. This allows fast reconfiguration (and in principle dynamic reconfigurability).
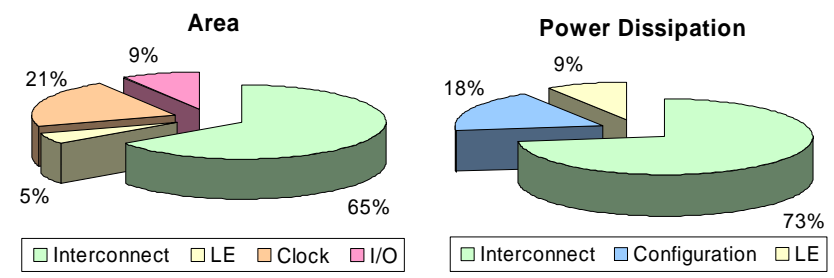

Fig. 3. FPGA area- and power breakdown.

To relax the global interconnect requirements, several logic elements can be combined in so-called clusters that share a central connection box. Typically, a cluster consists of about ten LEs. As delays for signals travelling long distances on the FPGA can get significant since they have to pass several routing switches, it is a common technique to provide segmented interconnects with routing lines of different lengths. In this case, certain routing channels skip several routing switches before connecting to another RS.

The programmable interconnect features the highest contribution to area, power dissipation and delay times of an FPGA. Figure 3 shows an area- and power breakdown taken from Kusse et al. (1998) and George et al. (2001). The figures show that the highest optimisation potential can be expected from the interconnect architecture. It is therefore indispensable to analyse the specific interconnect requirements of the given application domain and hence design the appropriate eFPGA-architecture.

\section{Arithmetic-oriented eFPGAs}

Commercial FPGA components are optimised for a wide range of applications, as they are intended for universal use. Significant research has been conducted in the optimisation of these universal FPGAs, using benchmark circuits from different application domains (e.g. Betz et al., 1999). An analysis of the logic- and interconnect requirements of arithmeticdatapaths reveals that the architectural requirements differ significantly from irregular logic. In this contribution, an eFPGA-architecture and the corresponding structural elements have been tailored to an arithmetic-oriented application domain. Considering the interconnect requirements of different applications mapped to FPGAs, it is useful to consider the histogram of the lengths of allocated interconnections. Fig. 4 shows such a qualitative histogram of the allocated connections between logic elements. For irregular logic, the number of connections between the LEs decreases with the connection length L. Arithmetic-datapaths, however, have a very high locality, hence exposing a peak in the distribution for short connections. Only few lines of intermediate length are required, while some long connections, usually representing broadcast lines, are used.

To confirm this qualitative histogram, an investigation of the allocated routing resources in a commercial FPGA de- 


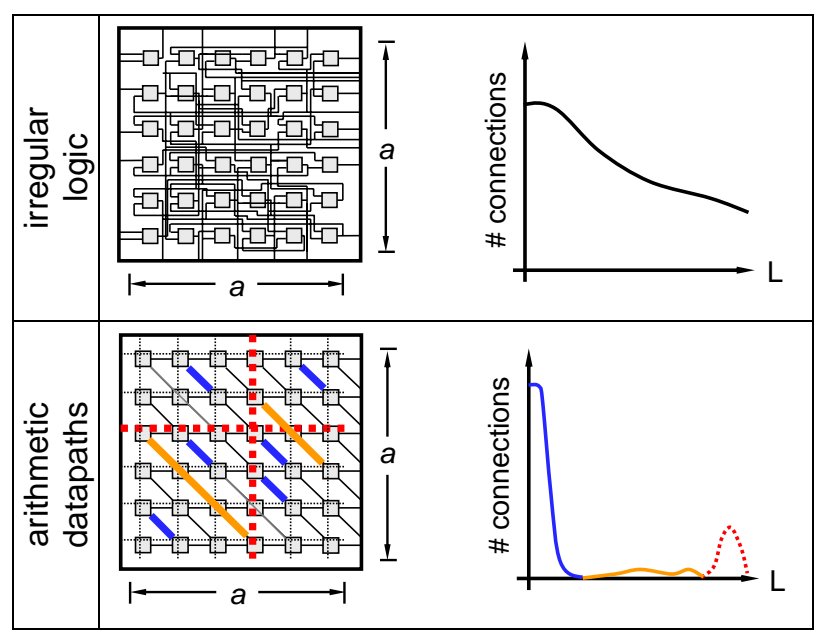

Fig. 4. Application domain specific routing requirements.

vice has been conducted. For this purpose, key components of a GPS-receiver (Global Positioning System) have been implemented on an Altera Stratix device (Kappen et al. 2006). Using the corresponding design software, the number of allocated routing resources according to their segment length has been determined. Figure 5 shows the results for two exemplary components, the correlator and the corresponding control logic. While the irregular control logic has a high fraction of intermediate segments and considerably less short and long segments, the correlator logic itself has a significant amount of direct nearest-neighbour connections. Since the overhead in the interconnect architecture of today's FPGAs is mainly due to their high flexibility, a considerable reduction of this overhead can be expected if the architecture is tailored to this application domain.

A further reduction can be achieved by carefully designing the logic elements to preserve the communication locality of arithmetic datapaths. In order to relax the interconnect requirements, appropriate dedicated logic and connections are provided in the LEs. A basic example is the use of dedicated carry-chains in commercial FPGAs. Direct connections between the LEs enable the use of adders with no additional routing involved in the carry-propagation.

Additional optimisation can be achieved if the LEs are designed such that frequently used basic operations of typical datapaths are directly mapped to one LE or LE cluster respectively. An example of optimising the LE architecture in that sense is to provide the possibility of realising a gated full-addition efficiently within one LE, as gated full-addition is a common part of many arithmetic-oriented applications (multiply, etc.). In the same way, other dedicated logic and dedicated interconnect can be employed to improve the efficiency of the architecture. For example, while typical logic elements feature one register, arithmetic datapaths with extensive pipelining require significantly more registers per LE.
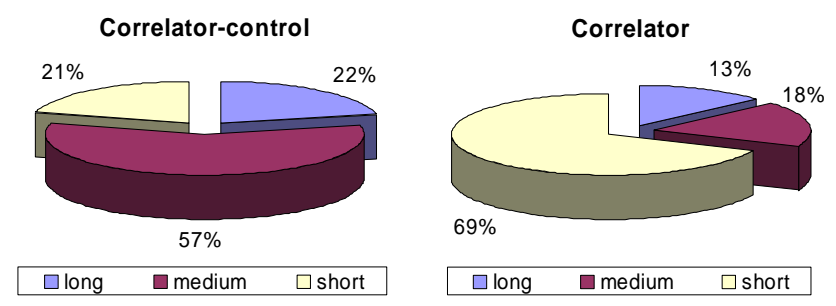

Fig. 5. Allocated interconnect lengths for irregular logic and arithmetic.

Table 1. Exemplary architectural parameters.

\begin{tabular}{ll}
\hline LE & $\begin{array}{l}\text { no. of in- /outputs } \\
\text { amount of dedicated logic }\end{array}$ \\
\hline Cluster & $\begin{array}{l}\text { no. and alignment of LEs } \\
\text { no. of in- /outputs }\end{array}$ \\
\hline Interconnect & $\begin{array}{l}\text { no. of hierarchy levels } \\
\text { size of routing channels } \\
\text { segmentation of routing channels } \\
\text { flexibility }\end{array}$ \\
\hline Configuration & $\begin{array}{l}\text { no. of parallel configuration sets } \\
\text { no. of shared configuration sets }\end{array}$ \\
\hline Others & granularity of supply and clock domains \\
\hline
\end{tabular}

\section{Parametrisable eFPGA-architectures}

In the following section, the design flow applied in this contribution is presented. This flow is based on an architecture template of the eFPGA with adjustable parameters. From this template, physically optimised realisations of the macro are created.

\subsection{Layout automation and parameters}

The basic design concept is based on generic architecture templates which are parametrisable in a wide range and a couple of physically optimised so-called leafcells. An automatic layout generation using a flexible datapath generator as described in (Weiss et al. 2001) is used to generate the layout of the eFPGA-macro. Table 1 lists some exemplary parameters influencing the basic structural elements of an eFPGAarchitecture.

These parameters feature strong interdependencies. For example, the architecture of the LE and the number of in- and outputs have a strong impact on the interconnect architecture.

In the present work, the design of a physically optimised layout for a parametrisable architecture is based on the following steps:

An architecture template has been conceived and realised in form of a generic structural description. A small set of key components like logic elements and switch points used for 


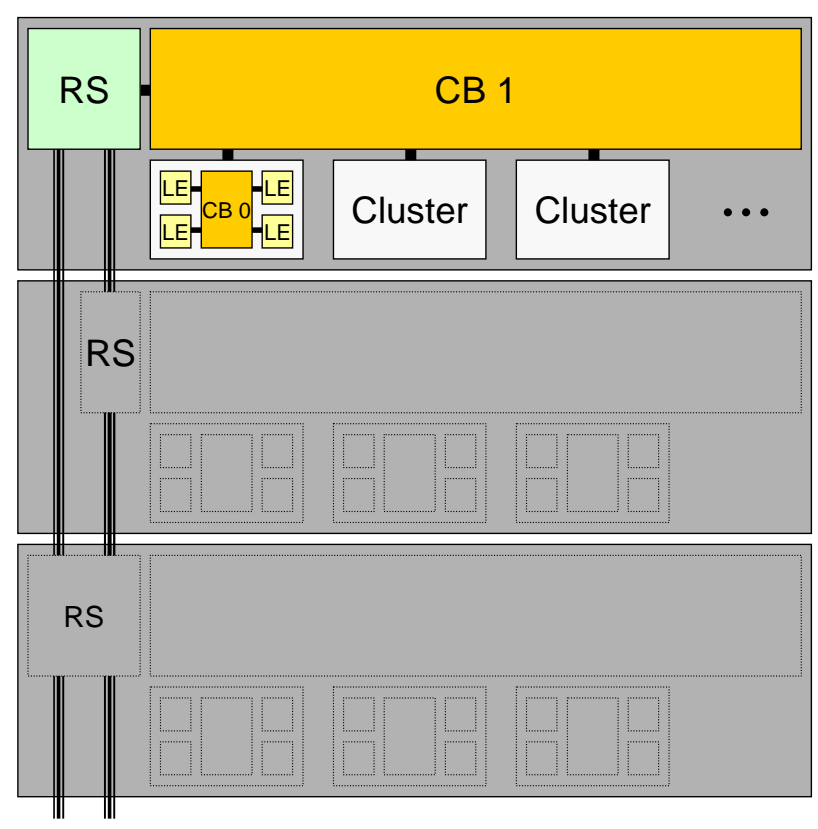

Fig. 6. Hierarchical reference architecture.

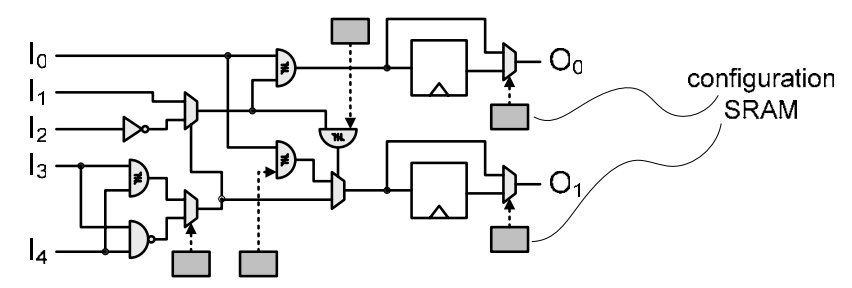

Fig. 7. LE of reference architecture.

routing switches has been designed. After adjusting all required parameters of the architecture template, a physically optimised macro is generated automatically applying the datapath generator. After the netlist extraction of this macro and setting up the simulation environment, a detailed timing and power analysis can be performed. If design constraints are not met, parameters can be easily adapted within the generic description and the macro generation process can be initiated again. The cycle time of adjusting parameters and acquiring cost and performance values depends on the complexity of the target macro.

\subsection{Reference architecture}

The first architecture template which has been realised serves as reference (Fig. 6). The interconnect architecture of this reference is based on the interconnect structure of modern commercial devices (Website Altera, Website Xilinx). A local connection box (CB 0 in Fig. 6) provides the connection between LEs arranged as clusters and the global connection box (CB 1 in Fig. 6). The global CB provides the connection to the global routing lines. Routing of signals coming from

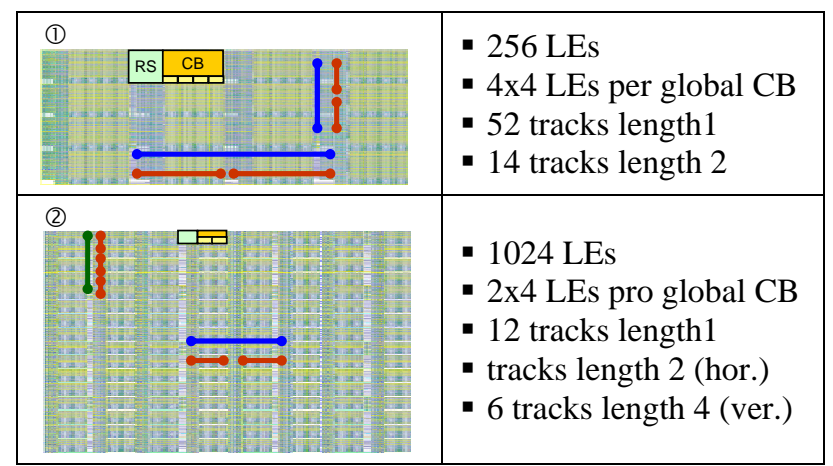

Fig. 8. Reference architecture macros and corresponding parameter settings.

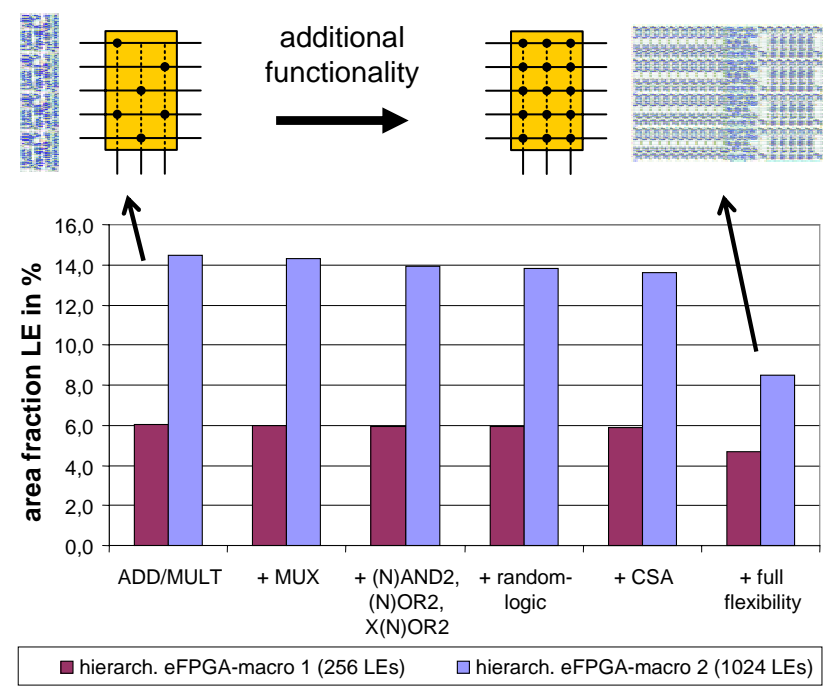

Fig. 9. Interrelation of flexibility and area within $\mathrm{CBO}$.

the global connection lines is executed by the RS. There are two types of routing switches: the first types enables regular vertical and horizontal routing between neighbouring RS, the second type is enhanced and offers the possibility of segmented routing for certain lines. Adaptable parameters of the corresponding architecture template are for example the number of routing tracks, the number of clusters per $C B$ and the length of interconnect lines for the segmented routing.

The LE architecture unlike commercial devices has been designed for arithmetic-oriented applications and is based on dedicated logic without employing LUTs (Fig. 7). Gated full-addition, add-compare-select and other arithmetic operations can be implemented very efficiently. In particular, the second register allows for efficient implementation of pipelined datapaths. The logic complexity of the LE is smaller than for a LUT with the same number of inputs. The number of SRAM-cells and hence overall silicon area required for this LE is much smaller than a LUT-based solution. 


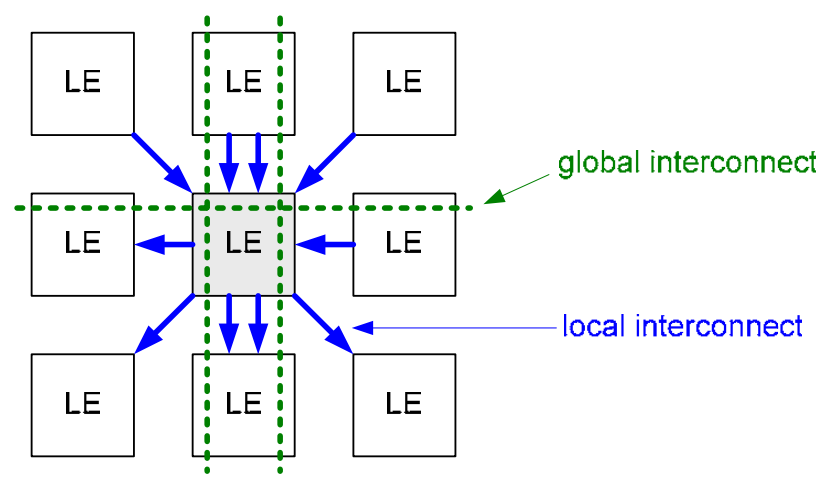

Fig. 10. Local and broadcast-interconnect.

Applying the design flow mentioned above, several macros with different parameter settings have been designed in a $180 \mathrm{~nm}$-technology. Two exemplary macros featuring different cluster sizes, different number of LEs per macro and different number and segmentation of routing tracks are depicted in Fig. 8.

For the local CB (CB0 in Fig. 6) a detailed analysis of flexibility requirements has been conducted using a variety of exemplary basic operations. Fig. 9 shows the area fraction of the LEs proportional to the complete macro for different levels of connection box flexibility. The flexibility of the complete architecture is determined by the number of possible connections within a CB. At first, the interconnect of the local CB has been designed for simple arithmetic datapaths (e.g. gated full addition, carry-ripple addition etc.). This means that only those connections are realised which are required for ADD/MULT operations. Such a restricted $\mathrm{CB}$ would mean that other types of operations cannot be performed with this $\mathrm{CB}$ configuration. In the next steps, the interconnect structure has been enhanced in a way that multiplexer functionality and randomised logic can be implemented (step 2, 3 and 4).

In the following step, more complex arithmetic datapaths have been included (e.g. carry-save arithmetic, CSA). The reference point in this depiction corresponds to the full flexible solution (rightmost bars). Above the diagram, the according layouts of the $\mathrm{CB}$, which are created automatically, are shown. The area fraction of the LEs is directly influenced by the routing parameters described above. Macro 1 includes more global routing tracks. Therefore, this solution features a higher flexibility than macro 2 which has got a more restricted routing architecture. The area of the LE and the local $\mathrm{CB}$ is the same for both macros.

For this first architecture template, the routing architecture is still the dominating factor in terms of area, timing and power consumption. Using the design methodology (see Sect. 4.1) established with the reference architecture, an eFPGA-architecture tailored for an arithmetic-oriented application domain has been developed.

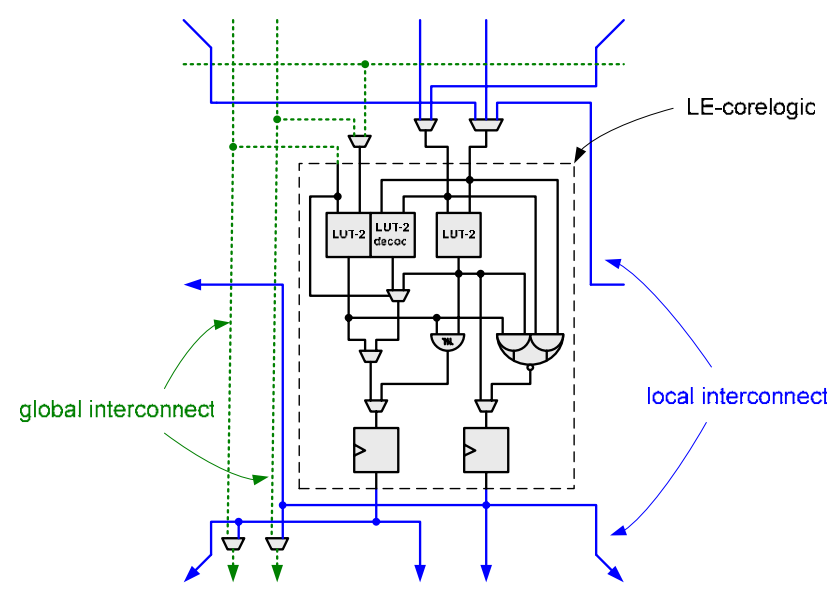

Fig. 11. Arithmetic-oriented LE.

\subsection{Arithmetic-oriented eFPGA-architecture}

The requirements of arithmetic-oriented datapaths have been addressed by the eFPGA architecture described in the following. The architecture uses arithmetic-oriented LEs that are connected as two-dimensional clusters, reflecting the typical orientation of processing elements in filters and multipliers. The LEs are connected with a directional nearest-neighbour interconnect. Rather than providing random connections to all surrounding neighbours, this structure reflects the typical nature of arithmetic-oriented datapaths featuring a preferred data flow direction. In this architecture, a typical data flow from top to bottom and right to left is assumed. Diagonal connections are provided in both left- and right-downwards direction to allow an efficient implementation of both rightand left-shift-operations. For efficient mapping of datapaths with arbitrary wordlengths, local connections are also provided across cluster borders. Signals from the global interconnect, typically representing operands in datapaths, are distributed along the LEs inside a cluster as so-called broadcast lines. This is in contrast to the highly flexible (and large) connection boxes in standard FPGAs that allow each LE to access global routing resources. Fig. 10 illustrates this data flow oriented interconnect.

The logic element used in the arithmetic-oriented eFPGA is depicted in Fig. 11. The core logic inside the dashed line consists of two lookup-tables with two inputs each, dedicated sum- and carry-logic and two latches.

One LUT-2 can be configured as partial-product gate using the global inputs to enable a direct mapping of arraymultiplier-based structures to the eFPGA. In addition, the two lookup-tables can be combined to a single LUT with three inputs (LUT-3). Dedicated multiplexers allow to connect the outputs of an LE to the broadcast-lines. The use of two latches per LE enables efficient pipelining of array-based datapaths. Rather than using complete registers, small transmission-gate-based latches reduce the implementation costs. 


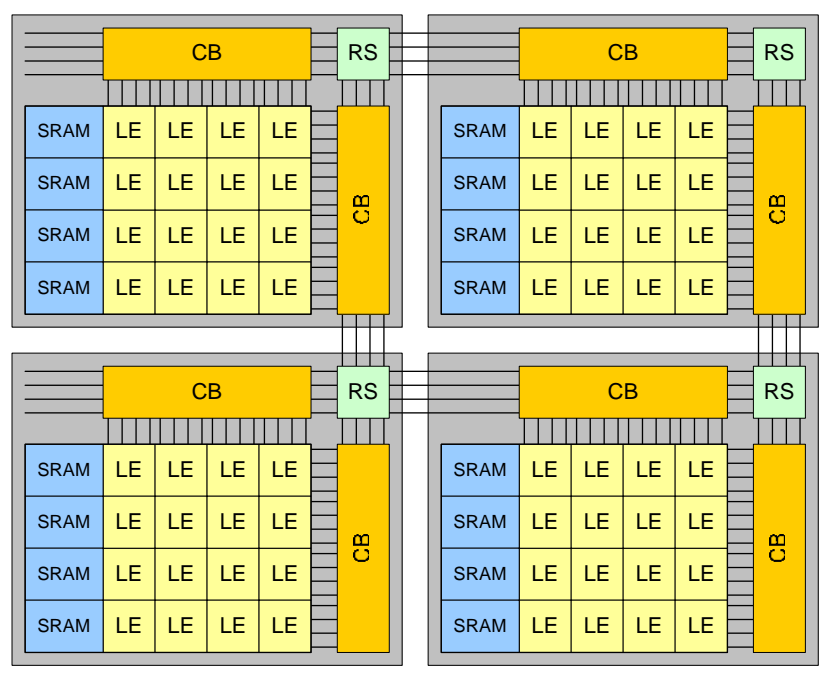

Fig. 12. Arithmetic-oriented architecture template.

The regularity and the typical wordlengths of arithmetic datapaths allow the shared use of configuration information, as for example presented in Ye et al., 2003). In this case, several logic elements inside a cluster use the same configuration bits. As the SRAMs storing the configuration typically amount for about half the area of a logic element, significant area-reductions can be achieved by choosing the appropriate granularity for the eFPGA-architecture. The same concept is applied to the global interconnect lines, thus reducing the overhead of the costly interconnect architecture. The overall architecture is shown in Fig. 12.

An according eFPGA-macro was designed in a $130 \mathrm{~nm}$ technology using a cluster size of $4 \times 4$ and a configuration granularity of four. The global interconnect provides only 16 tracks, as the communication in arithmetic-oriented datapaths is mainly based on local connections as shown before. A layout of four complete clusters with configuration blocks, CBs and RSs is shown in Fig. 13.

As can be seen from the layout, the area of the macro is dominated by the logic elements, while the area required by the global interconnect could be reduced significantly. The area fraction of the LE for the arithmetic-oriented eFPGA is $40 \%$, compared to less than $10 \%$ in typical standard FPGAs.

Figure 14 illustrates the LE-density (number of logic elements per $\mathrm{mm}^{2}$ ) for different (e)FPGA-architectures. It compares commercial standard FPGAs like the Altera APEX 20K (Website Altera) or the M2000 FlexEOS-eFPGA (Website M2000) as well as an exemplary research project, the Chimaera-architecture (Hauck et al. 2004) with the eFPGA-macros presented in this paper. Also, a basic islandstyle architecture as described in Fig. 2 is considered. As expected, the reference macro 1 has an LE-density similar to that of the APEX 20K, while reference macro 2 achieves a higher density due to the reduced number of routing tracks.

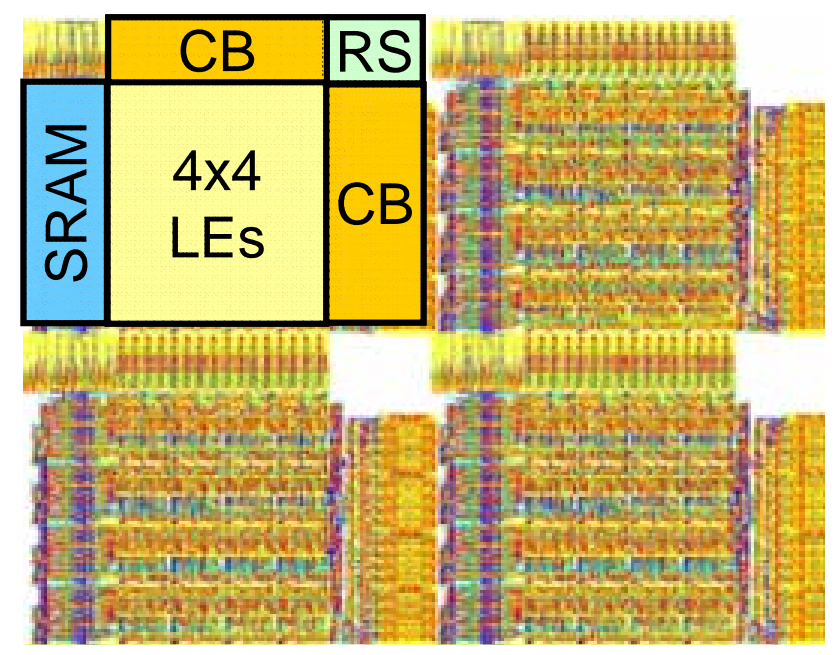

Fig. 13. Layout of arithmetic-oriented eFPGA.

Both the Chimaera-architecture and the commercial FlexEOS show a significant improvement compared to the discrete standard FPGA.

The arithmetic-oriented eFPGA presented in this paper achieves an LE-density that is one order of magnitude higher than for standard FPGAs. This comes at the cost of reduced flexibility when mapping arbitrary, irregular logic. Mapping irregular logic to this architecture would lead to losses in efficiency due to misalignments in data flow, inefficient usage of shared configurations and insufficient functional complexity on LE-level. However, for arithmetic-oriented applications the functional complexity of the LEs is equivalent to LE-complexities in standard FPGAs (i.e. a similar number of LEs is required to map a certain function to either a standard- or the arithmetic-oriented eFPGA). Hence, the area efficiency of arithmetic-datapaths can be improved by as much as an order of magnitude with the present architecture.

\section{Conclusion}

In this paper, an architecture template based designmethodology for domain specific eFPGAs has been presented. Based on the results of an exhaustive analysis of routing-, functionality- and regularity requirements of arithmetic-oriented applications, several architectural optimisations have been applied. The parametrisable template enables systematic optimisations according to the requirements of an application domain. Based on an analysis of arithmetic-oriented applications like filters and correlators, a novel eFPGA-architecture was presented. For this architecture, an area reduction of one order of magnitude compared to common FPGA-architectures was achieved. 


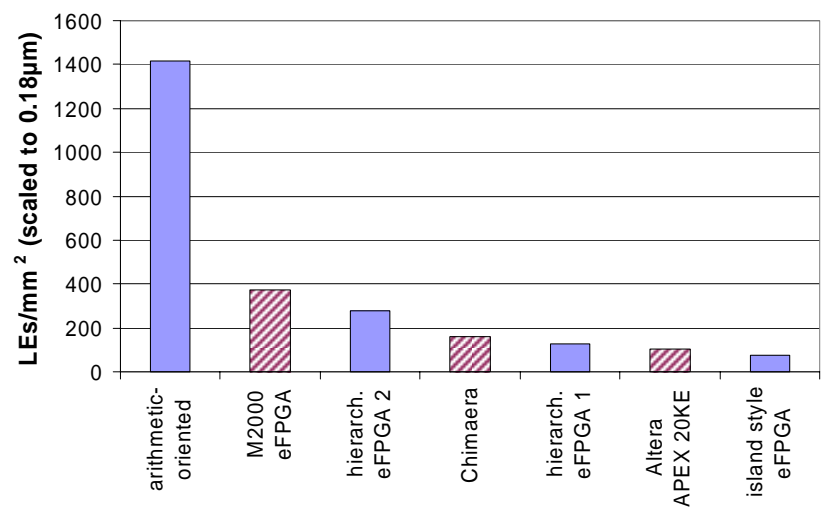

Fig. 14. LE-density for different eFPGA-architectures.

\section{References}

Betz, V., Rose, J. and Marquardt, A.: Architecture and CAD for Deep-Submicron FPGAs, Kluwer International Series in Engineering and Computer Science, ISBN 0-7923-8460-1, 1999.

Brown, S. and Rose, J.: FPGA and CLPD architectures: A tutorial, IEEE Design and Test of Computers, 13, 42-57, 1996.

George, V. and Rabaey, J. M.: Low Energy FPGAs - Architecture and Design, Kluwer International Series in Engineering and
Computer Science, 2001.

Hauck, S., Fry, T. W., Hosler, M. M., and Kao, J. P.: The Chimaera Reconfigurable Unit, IEEE Trans. on VLSI Systems, 12, 206217, 2004.

Kappen, G. and Noll, T. G.: Application Specific Instruction Processor Based Implementation of a GNSS Receiver on an FPGA, accepted for IEEE DATE, 2006.

Kusse, E. and Rabaey, J.: Low-energy embedded FPGA structures, Symp. on Low Power Electronics and Design (IEEE/ACM), 155-160, 1998.

Leijten-Nowak, K. and van Meerbergen, J. L.: An FPGA Architecture with Enhanced Datapath Functionality, Proc. FPGA '03, 194-204, 2003.

Neumann, B., Blume, H., Feldkämper, H., and Noll, T. G.: Embedded FPGAs für Multimedia-Applikationen, Proceedings ITGFachtagung "Elektronische Medien”, Dortmund, 147-152, 2003 (in german).

Website Altera: http://www.altera.com

Website M2000: http://www.m2000.fr

Website Xilinx: http://www.xilinx.com

Weiss, O., Gansen, M., and Noll, T. G.: A flexible Datapath Generator for Physical Oriented Design, Proceedings ESSCIRC, 408411, 2001.

Ye, A., Rose, J., and Lewis, D.: Architecture of Datapath-Oriented Coarse-Grain Logic and Routing for FPGAs, IEEE CICC, 6164, 2003. 